\title{
Evaluación de atención, memoria y flexibilidad cognitiva en niños bilingües
}

Yira Marieta Castro-Castiblanco

orcid.org/00oo-0001-9387-349X

Institución Universitaria Politécnico

Grancolombiano, Colombia

ycastroc@poligran.edu.co
Juan Bernardo Zuluaga-Valencia

orcid.org/00oo-0001-6228-8087

Universidad de Manizales, Colombia juanb@umanizales.edu.co

\section{Resumen}

Este artículo pretende identificar el impacto de la exposición temprana a una segunda lengua sobre algunas de las funciones ejecutivas, en relación con el tiempo de exposición y sexo de los participantes. El diseño metodológico es de tipo descriptivo, con alcance correlacional cuasi experimental, tipo caso control. La muestra es no probabilística, conformada por 30 niños en edades entre 5 y 6 años, en contexto escolarizado urbano. Para establecer el perfil cognitivo de los participantes se tomaron en cuenta como instrumentos la evaluación neuropsicológica infantil (ENI) y la escala WISC III abreviada (cubos y vocabulario). Se evidenció desempeño de los niños bilingües en memoria auditiva verbal; en atención visual y memoria de trabajo, los resultados son relativamente altos. En flexibilidad cognitiva, no se evidencian diferencias significativas para ambos grupos (bilingües y no bilingües). Encontramos diferencias entre niños y niñas: en memoria verbal auditiva y atención visual, las niñas muestran mejores desempeños, mientras que los niños obtuvieron mejores desempeños en las pruebas de memoria de trabajo. Por último, no encontramos correlación estadisticamente significativa entre coeficiente intelectual y resultados de la ENI.

\section{Palabras clave (fuente: tesauro de la Unesco)}

Bilingüismo; desarrollo infantil; desarrollo del niño; educación infantil; evaluación de aprendizajes; Bogotá; Colombia.

Recepción: 14/05/2018 | Envío a pares: 24/04/2019 | Aceptación por pares: 01/05/2019 | Aprobación: 13/05/2019 


\title{
Assessment of Attention, Memory and Cognitive Flexibility in Bilingual Children
}

\begin{abstract}
The article intends to identify the impact of early exposure to a second language on some executive functions in relation to exposure time and sex of participants. The methodological design is descriptive with a control case-type, quasi-experimental, correlational scope. The sample was used non-probabilistic, made up of 30 children aged between 5-6 from urban schools in Bogotá, Colombia. In order to determine the cognitive profile of participants, the child neuropsychological assessment (CNA) and the WISC III short form (cubes and vocabulary) were considered. These showed high performance of bilinguals in verbal auditory memory, while relatively high in visual attention and working memory. No significant differences were identified in cognitive flexibility between both groups (bilingual and non-bilingual). Differences were found between girls and boys; girls had higher performance in auditory verbal memory and visual attention, while boys performed better in working memory tests. Finally, no statistically significant correlation was established between intelligence quotient and the ENI results.
\end{abstract}

\section{Keywords (Source: Unesco Thesaurus)}

Bilingualism; child development; child education; learning assessment; Bogota; Colombia. 


\section{Avaliação da atenção, da memória e da flexibilidade cognitiva em crianças bilíngues}

\section{Resumo}

Este artigo pretende identificar o impacto da exposição precoce a uma segunda língua sobre algumas das funções executivas em relação ao tempo de exposição e ao sexo dos participantes. O desenho metodológico é de tipo descritivo, com alcance correlacional quase experimental tipo caso de controle. A amostra é não probabilística, conformada por 30 crianças, entre 5 e 6 anos, em contexto escolarizado urbano. Para estabelecer o perfil cognitivo dos participantes, foram utilizadas como instrumentos a avaliação neuropsicológica infantil (ANI) e a escala WISC III abreviada (cubos e vocabulário). Assim, ficou evidenciado o desempenho dos bilíngues em memória auditiva verbal; em atenção visual e memória de trabalho, os resultados são relativamente altos. Em flexibilidade cognitiva, não foram evidenciadas diferenças significativas para ambos os grupos (bilingues e não bilíngues). Verificaram-se diferenças entre meninos e meninas: em memória verbal auditiva e atenção visual, as meninas mostraram melhores desempenhos, enquanto os meninos obtiveram melhores desempenhos nos testes de memória de trabalho. Por último, não constatamos correlação estatisticamente significativa entre coeficiente intelectual e resultados da ANI.

\section{Palavras-chave (Fonte: tesauro da Unesco)}

Bilinguismo; desenvolvimento infantil; educação infantil; avaliação da aprendizagem; Bogotá; Colômbia 


\section{Introducción}

La mayor parte de los efectos documentados de la exposición al bilingüismo tienen que ver con los procesos de control atencional y desarrollo de las funciones ejecutivas, fluidez verbal y conciencia fonológica; no obstante, los resultados no son consistentes respecto al tiempo de exposición mínimo requerido para que en los aprendices de una segunda lengua ello surta efecto, cuestión a la cual pretende aportar la presente investigación (Bialystok y Feng, 2009).

Existen otros factores que influencian el desarrollo de las lenguas, tales como el coeficiente intelectual, componentes de orden genético, factores contextuales, de estimulación e incluso pedagógicos que pueden generar diferenciales en la manera en que se establece el proceso. No es lo mismo aprender dos lenguas desde el inicio de la vida y de manera simultánea que aprender la lengua materna o primera (L1) y, con posterioridad, una segunda lengua (L2) (Jung y López, 2003).

Los estudios con población colombiana que describen los efectos del bilingüismo en la infancia temprana son limitados, ya que la mayor parte se centran en los efectos posteriores sobre procesos cognitivos (Arango, Puerta, Montoya y Sánchez, 2013; Ardila, 2010; Fandiño, Bermúdez y Lugo 2012: Martínez y Henao, 2006; Mejía, López y Peña, 2011; Sánchez, 2013).

\section{Marco teórico}

\section{Proceso de adquisición de segunda lengua}

La definición de bilingüismo implica la mayor proficiencia posible o adecuado nivel de desempeño que tenga el individuo en cada una de las competencias del lenguaje (lectura, escritura y habla) para las dos lenguas, independientemente de si han sido adquiridas de manera simultánea o asincrónica; sin embargo, la total proficiencia en dos idiomas es más bien escasa, teniendo en cuenta que es poco probable su uso en contextos socioculturales con la misma intensidad y frecuencia, lo cual apunta más hacia la preponderancia de una lengua sobre la otra (el sujeto lo es más en una que en otra) (Jung y López, 2003). La adquisición de la L2, por su parte, surte un proceso en el cual las habilidades de escucha y habla se empiezan a desarrollar casi en su totalidad aproximadamente a los 3 años de exposición, mientras que las de lectoescritura empieza a establecerse a los 5 años de exposición en contextos académicos (Cummins, 2001).

Silva y Corvalán (citados por Jung y López, 2003) evidencian que los bilingües tempranos son proficientes en ambos idiomas, ya que adquieren unos principios de "gramática universal"; en contraste, los bilingües tardíos requieren de una etapa previa de consolidación de la L1, la cual no se ve afectada por la aparición posterior de una L2, pues se mantienen independientes. Sin embargo, si la L1 no se ha consolidado pueden existir trasferencias de $L_{1}$ a $L 2$, sobre todo si la primera tiene mayor uso.

Existe una estrecha relación entre los periodos sensibles del desarrollo y el aprendizaje de una L2. En el aprendizaje tardío de una segunda lengua, a mayor tiempo de inicio se dará menor eficacia o se requerirá mayor esfuerzo para obtenerla, incluso la motivación para el aprendizaje decrece con cada año de edad y las primeras experiencias que se tienen con la L1 determinan el éxito con la L2 (Janciauskas y Chang, 2017).

Para el bilingüismo tardío en contextos pedagógicos, es importante tener en cuenta la definición de educación bilingüe como vehículo de aprendizaje, respecto de la cual Fishman afirma que comprende "un sistema de enseñanza en el cual, en un momento variable y durante un tiempo y en proporciones variables, simultánea o consecutivamente, se da la instrucción al menos en dos lenguas, de las cuales una es la primera lengua del alumno" (1976, citado por Sánchez y Tembleque, 1986, p. 5). Además se requiere establecer un lazo afectivo con ambas lenguas debido a que "una lengua es una Weltan- 
schauung, una manera de encarar el mundo, de enfrentarse con él" (Balkan, 1979 citado por Smekal, 2014). La adquisición de una lengua o dos tiene un gran impacto en el desarrollo de la identidad y en el desarrollo de toda la personalidad (Bialystok, 2001; Balkan, 1979, citado por Smekal, 2014).

\section{Bilingüismo y cognición}

Respecto a los procesos cognitivos, se encuentra que, si se controla la variable socioeconómica, los bilingües muestran un mejor desempeño en las pruebas de coeficiente intelectual, y además se documentan correlaciones positivas entre bilingüismo y rendimiento académico (Cummins, 2001; Ardila, 2010), así como entre bilingüismo y desarrollo cognitivo, específicamente en funciones ejecutivas tales como inhibición, flexibilidad cognitiva y control atencional (Bialystok, 2004).

Respecto a las funciones ejecutivas, los niños bilingües superan en la mayoría de pruebas a los monolingües, beneficio que se da con mayor fuerza cuando se presenta mayor proficiencia en ambas lenguas. Además, la estimulación cognitiva ejercida por el bilingüismo es directamente proporcional al tiempo de exposición a un segundo idioma (Bialystok y Barac, 2012).

También existe una fuerte correlación entre control ejecutivo y estrato socioeconómico, siendo este determinante también a la hora de hablar de funciones ejecutivas, pues, independiente del bilingüismo, hay una fuerte y directa correlación entre estrato económico y desarrollo de dichas funciones, sumado a la crianza, los estilos parentales y la capacidad de inhibición o autocontrol (Barac y Bialystok, 2012).

En cuanto a los tiempos respecto de los cuales se empiezan a evidenciar beneficios en el desarrollo de las funciones ejecutivas, se encuentra que desde los 4 a 5 años de edad los niños bilingües poseen mejor ejecución en tareas de clasificación de tarjetas (dimensional change card sort), la cual se va incrementando a medida que se consolidan los dos idiomas, lo que da indicios sobre desarrollos importantes que suceden durante el periodo preescolar (Carlson, 2005; Zelazo y Müller, 2002; Bialystok, Craig, Klein y Viswanathan, 2004; citados por PoulinDubois, Blaye, Coutya y Bialystok, 2011).

Figura 1. Proficiencia y edad de adquisición de una segunda lengua

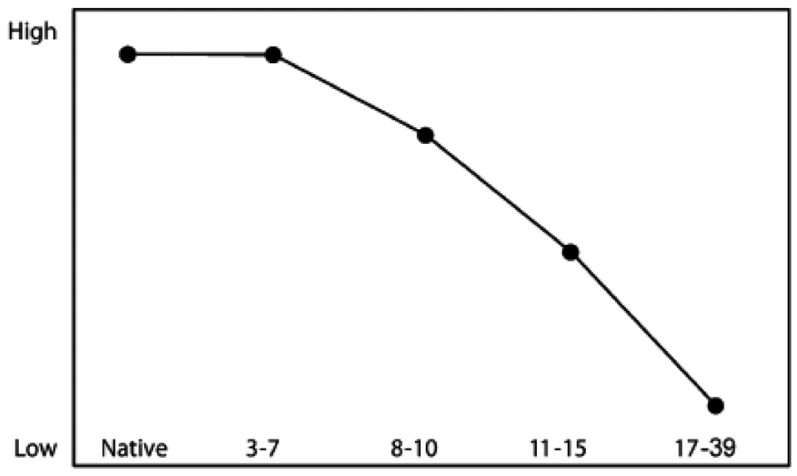

Fuente: tomado de Kuhl (2010).

Otro de los grandes impactos del bilingüismo tiene que ver con la inhibición y el control atencional, ya que se basa en la necesidad de supervisar la atención a la lengua que se está ejecutando en el contexto y manteniendo bajo control la posible interferencia de la que no se encuentra en uso (Calvo y Bialystok, 2014). Por otra parte, existe una clara correlación entre los procesos de atención visual y el bilingüismo, ya que, cuando se adquiere una L2, las señales o inputs visuales hacen parte importante del proceso de aprendizaje del idioma, pues permiten a los aprendices inferir el mensaje que se les quiere transmitir y estimulan de forma importante la atención visual (eye tracking) (Cintrón y Ellis, 2016).

Los estudios sobre el impacto del bilingüismo en la memoria de trabajo, no son concluyentes en las fuentes revisadas. Algunos indican una diferencia en tareas de memoria a corto plazo con mejor desempeño en bilingües, mientras que otros muestran que el desempeño es mejor en relación con ciertas tareas específicas ligadas con la memoria de trabajo: como aquellas visoespaciales y de control atencional y atención visual y atención auditiva, 
como la prueba ENI de dígitos en progresión y regresión (Calvo, Ibáñez y García, 2016; Morales, Calvo y Bialystok, 2013; Gorman, 2012).

Fandiño, Bermúdez y Lugo (2012) resumen así las ventajas del bilingüismo:

1. La plasticidad del cerebro y del sistema nervioso favorece el aumento de conexiones neuronales, lo cual posibilita que el aprendizaje se realice de manera rápida y fácil.

2. La facilidad para la imitación, la adaptabilidad y la memorización facilitan el aprendizaje y la comunicación.

3. La flexibilidad de los hábitos lingüísticos de L1 conlleva una menor interferencia lingüística.

4. La mayor flexibilidad cognitiva y las habilidades de pensamiento creativo ejercen una influencia positiva en otras áreas: L1, matemáticas y sociales.

5. La mayor interacción con el profesor y los compañeros favorece el desarrollo de las destrezas orales.

\section{Bilingüismo y sexo}

Al parecer, la adquisición de la lengua materna está claramente diferenciada según el sexo, como lo demuestra la perspectiva sociocultural, en la cual se conciben los factores del entorno como cruciales en la manera en la cual se obtiene el dominio de la lengua; asimismo, otras teorías, más del orden biológico, proponen que juegan un papel fundamental en este aspecto las diferencias en la organización del cerebro para niños y niñas, el grado de maduración del sistema neuronal y las habilidades cognitivas (Jiménez, 2010).

Jiménez (2010) refiere que existen diferencias entre sexos en la ejecución de tareas cognitivas sobre todo en habilidades verbales y visoespaciales. Se ha encontrado que las niñas presentan por regla general una aparición más temprana de su lenguaje (entre los 14 y los 20 meses) y mayor éxito en el aprendizaje de una lengua, por cuanto logran: estructurar frases más largas y articular mejor, un vocabulario más amplio y la iniciativa para comenzar conversaciones con mayor frecuencia que los niños. Sin embargo, también se encuentra que en muchas oportunidades los resultados son sobrevalorados y, en otros estudios, son contradictorios. Jiménez cita diversos estudios (Macaulay, 1977; Graves y Koziol, 1971; Maccoby y Jackil, 1974) en los cuales no se han hallado diferencias significativas en el desarrollo lingüístico de las niñas o las diferencias no son significativas.

Y aunque Halpern (1992, citado por Jiménez, 2010) respalda las diferencias en desarrollo cerebral de niños y niñas, señala que aún no es suficiente como para afirmar que esto genera un desempeño superior de uno u otro sexo. Lo único que los estudios revelan con mayor certeza es que los niños son más proclives a presentar trastornos del lenguaje y que en definitiva las niñas poseen como factor protector una maduración más rápida de su sistema nervioso y una menor laterización (Macaulay, 1997; Lozano, 1995, en Jiménez).

\section{Bilingüismo y estrato socioeconómico}

Calvo y Bialystok (2014) evidencian que las condiciones ambientales pueden modificar la capacidad cognitiva; de esta manera, el lenguaje y las funciones ejecutivas son los dos sistemas neurocognitivos más influenciados por el nivel socioeconómico. Cabe señalar que existe, además, una alta correlación entre el nivel educativo de los padres y el desempeño cognitivo global de los niños, sobre todo en tareas que miden funciones ejecutivas, en donde la educación de los padres es el mejor predictor de desempeño de los niños. En las puntuaciones de coeficiente intelectual entre los grupos de alto y bajo nivel socioeconómico se encuentra documentada una diferencia que alcanza la desviación estándar. 
Calvo y Bialystok (2014) también han encontrado relaciones entre desempeño con puntajes superiores para bilingües de estrato socioeconómico más alto, en aspectos específicos de memoria de trabajo, control cognitivo e inhibición, mayores índices de eficacia atencional y de atención sostenida y visual, versus niños en condiciones económicas menos favorables, quienes además presentarían niveles inferiores en ambas habilidades del lenguaje (receptiva y expresiva), pero sin diferencias en cuanto a desempeño respecto a sistemas de procesamiento de la recompensa para ambos grupos. En conclusión, los efectos tanto del bilingüismo como del estrato socioeconómico parecen ser aditivos, lo cual implica que, si se dan ambas características, el desempeño es todavía mejor.

\section{Bilingüismo en Colombia}

Desde el punto de vista neuropsicológico, en un estudio para el contexto colombiano donde se relacionan funciones ejecutivas y se comparan grupos de niños bilingües y monolingües de estratos socioeconómicos 4 a 6 , se a plicó la prueba de clasificación de tarjetas de Wisconsin a una muestra de 32 sujetos del grado de preescolar. Se hallaron desempeños superiores en los niños bilingües, pero sin diferencias estadísticamente significativas y sin haber realizado una exploración de tipo neuropsicológico de mayor profundidad (Martínez y Henao, 2006).

Otro estudio, realizado por Villamizar y Guevara (2013 con adolescentes (15 bilingües y 20 monolingües) con un diseño de tipo no experimental correlacional, halló que existe una correlación positiva entre la inhibición y el bilingüismo, pero que no existía correlación entre la flexibilidad y el bilingüismo, resultados que se explican en el hecho de que los participantes del estudio tuvieron un bilingüismo tardío (posterior a los 5 años de edad) y que, para lograr identificar un efecto mayor del bilingüismo sobre las funciones ejecutivas, debe haber un bilingüismo temprano antes de los 5 años de edad. Respecto a la inhibición, se encuentra una correlación positiva debido al control atencional que se ejerce de manera constante al pasar de una lengua a otra.

Por último, un estudio de tipo empírico-analítico, descriptivo y trasversal (Mora, 2012), obtuvo una muestra de 51 niños de edades entre 7 y 12 años y concluyó que los estudiantes bilingües presentan mayor dependencia de campo en sus estilos cognitivos, sobre todo en edades más tempranas, con un incremento hacia la independencia de campo a medida que aumenta la edad, y también constató mayor tendencia a la dependencia de campo o sensibilidad al medio en mujeres, siendo los varones más independientes del campo: "En el área clínica es oportuno indagar a profundidad los estilos cognitivos con relación al desarrollo de la especialización hemisférica y desarrollo de funciones neuropsicológicas, a través de la aplicación de diversas pruebas neuropsicológicas en estos niños y niñas expuestos al bilingüismo" (p. 97).

Algunas de las mayores limitaciones de los estudios realizados tienen que ver con el poco control sobre la variable del coeficiente intelectual general, ya que no lo usan como criterio de selección de la población. Adicional a ello, los resultados tienden a ser poco concluyentes debido a los tamaños reducidos de las muestras poblacionales y, por otra parte, a la desigual proficiencia en las dos lenguas, tanto en el dominio como en el uso de la lengua en contexto.

\section{Método}

\section{Participantes}

La muestra de investigación estuvo conformada por 30 sujetos (14 niños y 16 niñas), todos escolarizados en los grados de transición y primero de primaria,

1 "La dependencia y la independencia de campo son dos de los estilos cognitivos que pueden manifestar los individuos. La dependencia del campo se caracteriza por la tendencia a percibir un fenómeno como un todo unitario, sin atender a las diferentes partes que lo integran; la independencia, por el contrario, consiste en la capacidad de aislar un determinado rasgo que se percibe como relevante, del contexto en el que se integra" (Instituto Cervantes, s.f.). 
pertenecientes a instituciones educativas privadas de la ciudad de Bogotá. La selección de la muestra se realizó de manera aleatoria, con un diseño de investigación de tipo no experimental, trasversal, con caso de control. En el grupo de bilingües, el $40 \%$ de los participantes fueron de sexo masculino, mientras que en el grupo de monolingües la representación masculina fue del 6o\%. En la Tabla 1 se presenta la media y la desviación estándar de la edad y el coeficiente intelectual (CI) total de los participantes.

Tabla 1. Media y desviación estándar de la edad y el Cl total, por caso y control

\begin{tabular}{|c|c|c|c|c|c|c|c|c|}
\hline \multicolumn{4}{|c|}{ Bilingües } & \multicolumn{4}{c|}{ Monolingües } \\
\hline V & M & DS & Mín. & Máx. & M & DS & Mín. & Máx. \\
\hline $\begin{array}{c}\text { Cl } \\
\text { total }\end{array}$ & 112,80 & 7,96 & 97,00 & 120,00 & 100,53 & 12,70 & 86,00 & 120,00 \\
\hline Edad & 6,07 & 0,19 & 5,92 & 6,50 & 6,39 & 0,35 & 5,92 & 6,92 \\
\hline
\end{tabular}

V: variable; M: media; DS: desviación estándar; Min.: valor mínimo; Máx.: valor máximo.

Fuente: elaboración propia.

En cuanto a la edad, el promedio de la de los monolingües fue $5 \%$ mayor que la edad promedio de los bilingües. Respecto al Cl total, el puntaje promedio de los bilingües fue 12,2\% mayor que el puntaje promedio de los monolingües. Los padres de estos niños aceptaron vincular de manera voluntaria a sus hijos como participantes, para lo cual firmaron consentimiento informado y además diligenciaron datos básicos como tipo de familia, estrato socioeconómico, ultimo nivel de escolaridad de los padres.

CRITERIOS de InClusión. Como criterio de selección de la muestra, todos los niños debían tener de 5 años 11 meses a 6 años 11 meses de edad, un Cl entre 85 y 115 según la escala de Wechsler de inteligencia para niños (Wechsler Intelligence Scale for Children - WISC III), determinado con la forma abreviada de las escalas de cubos y vocabulario, diseñada por Sattler (2003). Los niños bilingües debían haber estado por mínimo dos años recibiendo un promedio de 24 horas semanales de materias en inglés por dicho periodo. En cuanto al grupo de control, los participantes provenían de estratos 3 y 4 , sin exposición al bilingüismo, solo con conocimientos muy mínimos de inglés, criterios para ubicarlos en condición de monolingües.

\section{Instrumentos de evaluación}

Para la medición de las diferentes subpruebas de atención, memoria de trabajo, flexibilidad cognitiva se utilizó la evaluación neuropsicológica infantil (ENI) (Matute, Sanz, Gumá, Rosselli y Ardila, 2010) en las subpruebas de:

1. Memoria verbal auditiva: Lista de palabras, curva de memoria, recobro espontáneo, reconocimiento por claves, reconocimiento verbal auditivo.

2. Atención visual: Cancelación de dibujos, cancelación de letras.

3. Atención auditiva y memoria de trabajo: Dígitos en progresión, dígitos en regresión.

4. Flexibilidad cognitiva: Tarjetas de Wisconsin.

En la misma sesión se aplicó la escala abreviada de WISC III y las subpruebas de la ENI, que los niños tardaban un promedio de 45 minutos en completar, y ya que se tenía contacto con los niños de ambas poblaciones, se requería de mayor tiempo para entablar rapport.

\section{Procedimiento}

Primera fase: selección de la población según criterios de inclusión y revisión teórica de antecedentes; segunda fase: aplicación de pruebas para grupo de casos y grupo de control; tercera fase: análisis estadístico de la información; cuarta fase: retroalimentación de resultados a los padres y las instituciones educativas; quinta fase: elaboración de la discusión y conclusiones.

\section{Resultados}

El análisis de la información recolectada mediante las pruebas se llevó a cabo con el software de 
procesamiento estadístico SPSS versión 21. Para ello se tomó como variable independiente el grupo al que pertenecían los participantes, el cual se determinó con base en el criterio de inclusión, segmentando entre bilingües y monolingües. Como variables dependientes se analizaron los puntajes obtenidos por los participantes en las pruebas mencionadas. A continuación se presentan los principales resultados.

\section{Análisis bilingüe/monolingüe}

La Tabla 2 contiene el valor medio $(\bar{x})$ y la desviación estándar (DS) de cada una de las pruebas de memoria, atención y funciones ejecutivas, para cada grupo, que indican que la puntuación promedio fue mayor para la muestra de bilingües en las pruebas de memoria auditiva, atención visual y atención auditiva, con un menor grado de dispersión de los puntajes obtenidos, lo cual implica resultados más homogéneos para este grupo. No obstante, las diferencias fueron poco significativas en relación con el desempeño de ambos grupos en las tareas de flexibilidad cognitiva. Por último, se presenta la comparación de medias entre grupos efectuada mediante la prueba de $U$ de Mann-Whitney. Para la elección de dicha prueba se verificó la normalidad de los puntajes mediante la prueba de Shapiro-Wilk, determinando que no seguían una distribución normal (valor $p<0,05)$, motivo por el cual se utilizó una prueba no paramétrica.

Tabla 2. Media y desviación estándar de las pruebas de memoria auditiva, atención y flexibilidad cognitiva para bilingües y monolingües, diferencias porcentuales entre grupos y comparación de medias

\begin{tabular}{|c|c|c|c|c|c|c|c|c|}
\hline \multirow[b]{2}{*}{ Categorías } & \multirow[b]{2}{*}{ Variables } & \multicolumn{2}{|c|}{ Bilingüe } & \multicolumn{2}{|c|}{ Monolingüe } & \multirow{2}{*}{$\begin{array}{c}\text { \% diferencia } \\
\text { Bilingüe Vs. } \\
\text { Monolingüe }\end{array}$} & \multicolumn{2}{|c|}{ Comparación de medias } \\
\hline & & $\bar{x}$ & DS & $\overline{\mathbf{x}}$ & DS & & $\begin{array}{l}\text { U de Mann- } \\
\text { Whitney }\end{array}$ & Valor $p$ \\
\hline \multirow{4}{*}{$\begin{array}{l}\text { Memoria } \\
\text { auditiva }\end{array}$} & Curva de memoria & 21,7 & 3,9 & 17,5 & 4,9 & 24,4 & 58,0 & $0,023^{*}$ \\
\hline & Recobro espontáneo & 6,3 & 1,4 & 5,2 & 1,1 & 20,5 & 61,5 & $0,029^{*}$ \\
\hline & Recobro por claves & 7,3 & 1,6 & 5,5 & 2,0 & 34,1 & 53,0 & $0,012^{*}$ \\
\hline & $\begin{array}{l}\text { Reconocimiento verbal } \\
\text { auditivo }\end{array}$ & 16,5 & 1,9 & 14,9 & 2,6 & 10,8 & 64,0 & $0,041^{*}$ \\
\hline \multirow{2}{*}{$\begin{array}{l}\text { Atención } \\
\text { visual }\end{array}$} & Cancelación de dibujos & 17,5 & 5,5 & 10,1 & 4,3 & 72,4 & 32,5 & $0,001^{*}$ \\
\hline & Cancelación de letras & 14,3 & 3,8 & 11,4 & 4,6 & 25,1 & 75,5 & 0,123 \\
\hline \multirow{2}{*}{$\begin{array}{l}\text { Atención } \\
\text { auditiva }\end{array}$} & Dígitos en progresión & 5,1 & 1,2 & 3,2 & 1,0 & 58,3 & 25,5 & O,०००* \\
\hline & Dígitos en regresión & 2,5 & 0,5 & 1,6 & 0,8 & 58,3 & 42,5 & $0,001^{*}$ \\
\hline \multirow{4}{*}{$\begin{array}{c}\text { Flexibilidad } \\
\text { cognitiva }\end{array}$} & Respuestas correctas & 34,9 & 2,1 & 33,3 & 2,7 & 4,8 & 78,5 & 0,154 \\
\hline & Errores & 14,3 & 6,2 & 16,9 & 5,0 & $15,4^{a}$ & 84,0 & 0,236 \\
\hline & Categorías & 2,5 & 0,5 & 2,1 & 0,5 & 15,6 & 78,5 & 0,094 \\
\hline & Perseveraciones & 5,7 & 4,4 & 8,1 & 5,2 & $28,9^{a}$ & 86,0 & 0,270 \\
\hline
\end{tabular}

Fuente: elaboración propia. 


\section{Figura 2. Puntaje promedio en las pruebas monolingüe vs. bilingüe}

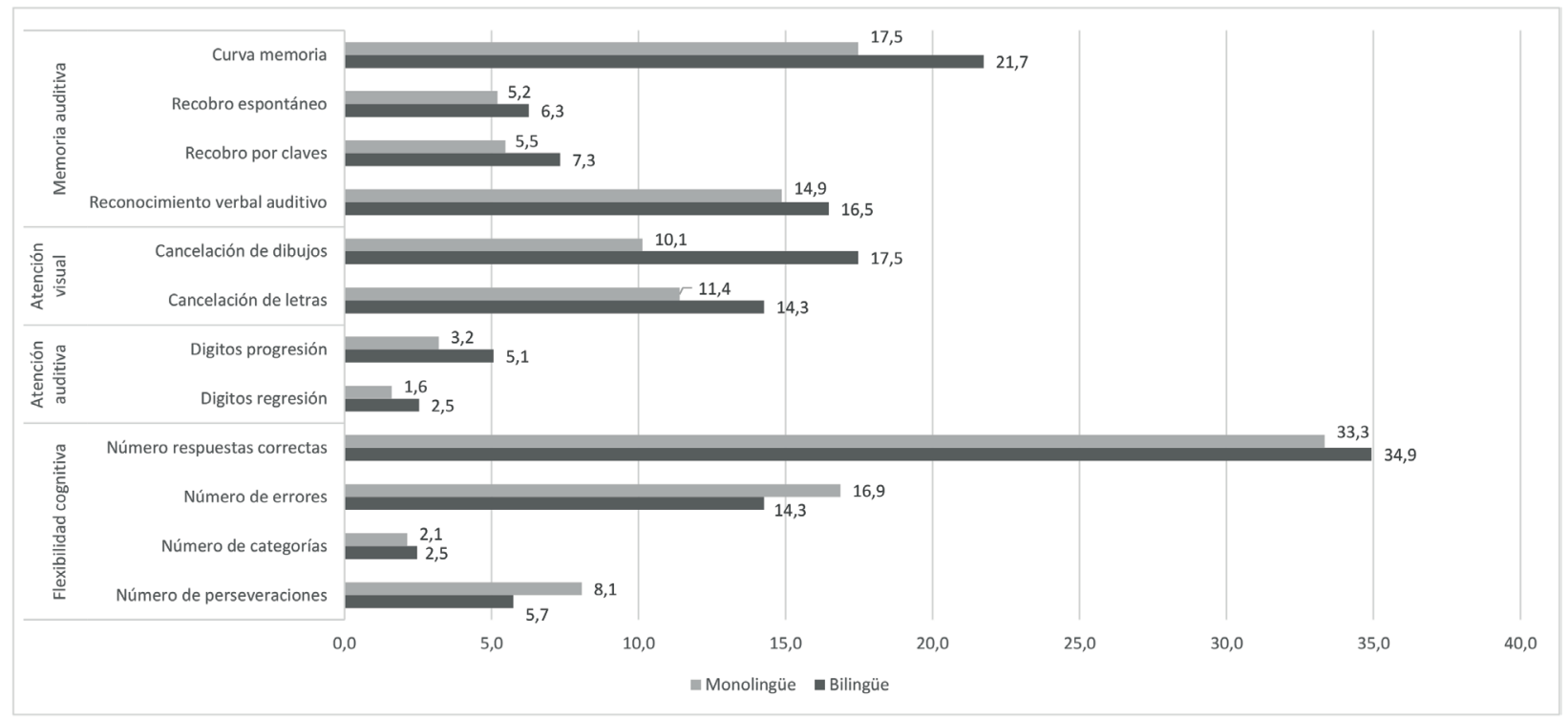

Fuente: elaboración propia.

En la memoria de auditiva, el grupo bilingüe tuvo mayor puntuación promedio en las cuatro pruebas aplicadas, lo que evidencia mayor diferencia porcentual entre la puntuación de grupos en el recobro por claves, con 34,1\%. Al comparar las medias de ambos grupos, se encontró que en todas las pruebas las diferencias eran estadísticamente significativas. En atención visual, también el grupo bilingüe tuvo mayor puntuación promedio en las dos pruebas, evidencia de una mayor diferencia porcentual entre la puntuación de grupos en la cancelación de dibujos, con un $72,4 \%$. Al comparar las medias de ambos grupos se encontró que solo existían diferencias estadísticamente significativas en la prueba de cancelación de dibujos. En atención auditiva, nuevamente el grupo bilingüe tuvo mayor puntuación promedio en las dos pruebas, y se evidenció igual diferencia porcentual entre la puntuación de grupos para ambas pruebas, con $58,3 \%$. Al comparar las medias de ambos grupos se encontró que existían diferencias estadísticamente significativas en las dos pruebas.
En cuanto a la flexibilidad cognitiva, el grupo bilingüe tuvo mayor puntuación promedio en el número de respuestas correctas y en el número de categorías, y hubo mayor diferencia porcentual entre la puntuación de grupos en el número de categorías, con $15,6 \%$. A su vez, el grupo monolingüe tuvo mayor puntuación promedio en el número de errores y en el número de perseveraciones, lo que arroja mayor diferencia porcentual entre la puntuación de grupos en el número de perseveraciones, con 28,9\%. Es importante aclarar que, en el número de errores y el número de perseverativas, mayor puntuación representa menor desempeño del participante.

\section{Análisis de las puntuaciones por sexo}

Se presenta un análisis que compara el desempeño entre participantes bilingües y monolingües, diferenciados por sexo femenino y masculino (Tabla 3/Figura 3), con el valor medio y la desviación estándar de cada una de las pruebas de memoria auditiva, atención y flexibilidad cognitiva para cada grupo, segmentado por sexo. Así mismo, se muestra el por- 
centaje de diferencia de los puntajes medios entre grupos para sexo femenino y masculino, estableciendo siempre como valor de referencia el puntaje del grupo de monolingüe, por lo cual los valores positivos indican que la puntuación media fue mayor para el grupo bilingüe y los valores negativos (marcado con la letra a) que la puntuación media fue mayor para el grupo monolingüe. Por último, se presenta la comparación de medias entre grupos por sexo, efectuada mediante la prueba de $U$ de Mann-Whitney.

Tabla 3. Media y desviación estándar de las pruebas de memoria auditiva, atención y flexibilidad cognitiva para bilingües y monolingües por sexo, diferencias porcentuales entre grupos por sexo y comparación de medias

\begin{tabular}{|c|c|c|c|c|c|c|c|c|c|c|c|c|c|c|c|}
\hline \multirow[b]{3}{*}{ Categorías } & \multirow[b]{3}{*}{ Variables } & \multicolumn{4}{|c|}{ Bilingüe } & \multicolumn{4}{|c|}{ Monolingüe } & \multirow{2}{*}{\multicolumn{2}{|c|}{$\begin{array}{l}\text { Diferencia \% } \\
\text { bilingüe vs. } \\
\text { monolingüe }\end{array}$}} & \multicolumn{4}{|c|}{ Comparación de medias } \\
\hline & & \multicolumn{2}{|c|}{ Femenino } & \multicolumn{2}{|c|}{ Masculino } & \multicolumn{2}{|c|}{ Femenino } & \multicolumn{2}{|c|}{ Masculino } & & & \multicolumn{2}{|c|}{ Femenino } & \multicolumn{2}{|c|}{ Masculino } \\
\hline & & $x$ & DS & $x$ & DS & $x$ & DS & $x$ & DS & Fem. & Masc. & $\begin{array}{c}\text { Ude } \\
\text { Mann- } \\
\text { Whitney }\end{array}$ & Valor $p$ & $\begin{array}{c}\text { Ude } \\
\text { Mann- } \\
\text { Whitney }\end{array}$ & Valor $\mathrm{p}$ \\
\hline \multirow{4}{*}{$\begin{array}{l}\text { Memoria } \\
\text { auditiva }\end{array}$} & Curva de memoria & 21,9 & 4,6 & 21,5 & 2,8 & 15,2 & 3,8 & 19,0 & 5,1 & 44,3 & 13,2 & 7,5 & $0,021^{*}$ & 19,5 & 0,373 \\
\hline & $\begin{array}{l}\text { Recobro } \\
\text { espontáneo }\end{array}$ & 6,4 & 1,6 & 6,0 & 1,1 & 4,7 & 0,8 & 5,6 & 1,1 & 38,1 & 8,0 & 9,0 & $0,030^{*}$ & 19,5 & 0,335 \\
\hline & Recobro por claves & 7,7 & 1,8 & 6,8 & 1,3 & 4,2 & 1,8 & 6,3 & 1,7 & 84,0 & 7,9 & 3,0 & $0,004^{*}$ & 22,5 & 0,585 \\
\hline & $\begin{array}{l}\text { Reconocimiento } \\
\text { verbal auditivo }\end{array}$ & 16,8 & 2,0 & 16,0 & 1,8 & 15,2 & 2,6 & 14,7 & 2,7 & 10,6 & 9,1 & 15,5 & 0,159 & 18,0 & 0,281 \\
\hline \multirow{2}{*}{$\begin{array}{c}\text { Atención } \\
\text { visual }\end{array}$} & $\begin{array}{l}\text { Cancelación de } \\
\text { dibujos }\end{array}$ & 17,7 & 5,7 & 17,2 & 5,7 & 8,3 & 3,4 & 11,3 & 4,6 & 112,0 & 51,5 & 4,5 & $0,008^{*}$ & 11,0 & 0,058 \\
\hline & $\begin{array}{l}\text { Cancelación de } \\
\text { letras }\end{array}$ & 13,6 & 4,1 & 15,3 & 3,4 & 9,7 & 4,2 & 12,6 & 4,8 & 40,2 & 22,1 & 13,5 & 0,109 & 18,5 & 0,310 \\
\hline \multirow{2}{*}{$\begin{array}{l}\text { Atención } \\
\text { auditiva }\end{array}$} & $\begin{array}{l}\text { Dígitos en } \\
\text { progresión }\end{array}$ & 4,8 & 1,1 & 5,5 & 1,2 & 3,7 & 1,0 & 2,9 & 0,9 & 30,3 & 90,4 & 13,0 & 0,086 & 1,0 & $0,002^{*}$ \\
\hline & $\begin{array}{l}\text { Dígitos en } \\
\text { regresión }\end{array}$ & 2,4 & 0,5 & 2,7 & 0,5 & 1,5 & 1,0 & 1,7 & 0,7 & 63,0 & 60,0 & 12,0 & 0,057 & 7,0 & $0,007^{*}$ \\
\hline \multirow{4}{*}{$\begin{array}{c}\text { Flexibilidad } \\
\text { cognitiva }\end{array}$} & $\begin{array}{l}\text { Respuestas } \\
\text { correctas }\end{array}$ & 35,0 & 2,4 & 34,8 & 1,6 & 33,5 & 2,8 & 33,2 & 2,8 & 4,5 & 4,8 & 18,5 & 0,313 & 17,5 & 0,256 \\
\hline & Errores & 15,4 & 6,3 & 12,5 & 6,0 & 19,3 & 2,8 & 15,2 & 5,6 & $20,1^{a}$ & $17,9^{\mathrm{a}}$ & 16,0 & 0,193 & 19,0 & 0,341 \\
\hline & Categorías & 2,3 & 0,5 & 2,7 & 0,5 & 1,8 & 0,4 & 2,3 & 0,5 & 27,3 & 14,3 & 15,0 & 0,068 & 18,0 & 0,221 \\
\hline & Perseveraciones & 6,9 & 4,5 & 4,0 & 4,1 & 10,5 & 6,9 & 6,4 & 3,3 & $34,4^{a}$ & $37,9^{a}$ & 19,5 & 0,376 & 15,0 & 0,154 \\
\hline
\end{tabular}

Fuente: elaboración propia. 
Figura 3. Puntaje promedio en las pruebas monolingüe vs. bilingüe, por sexo

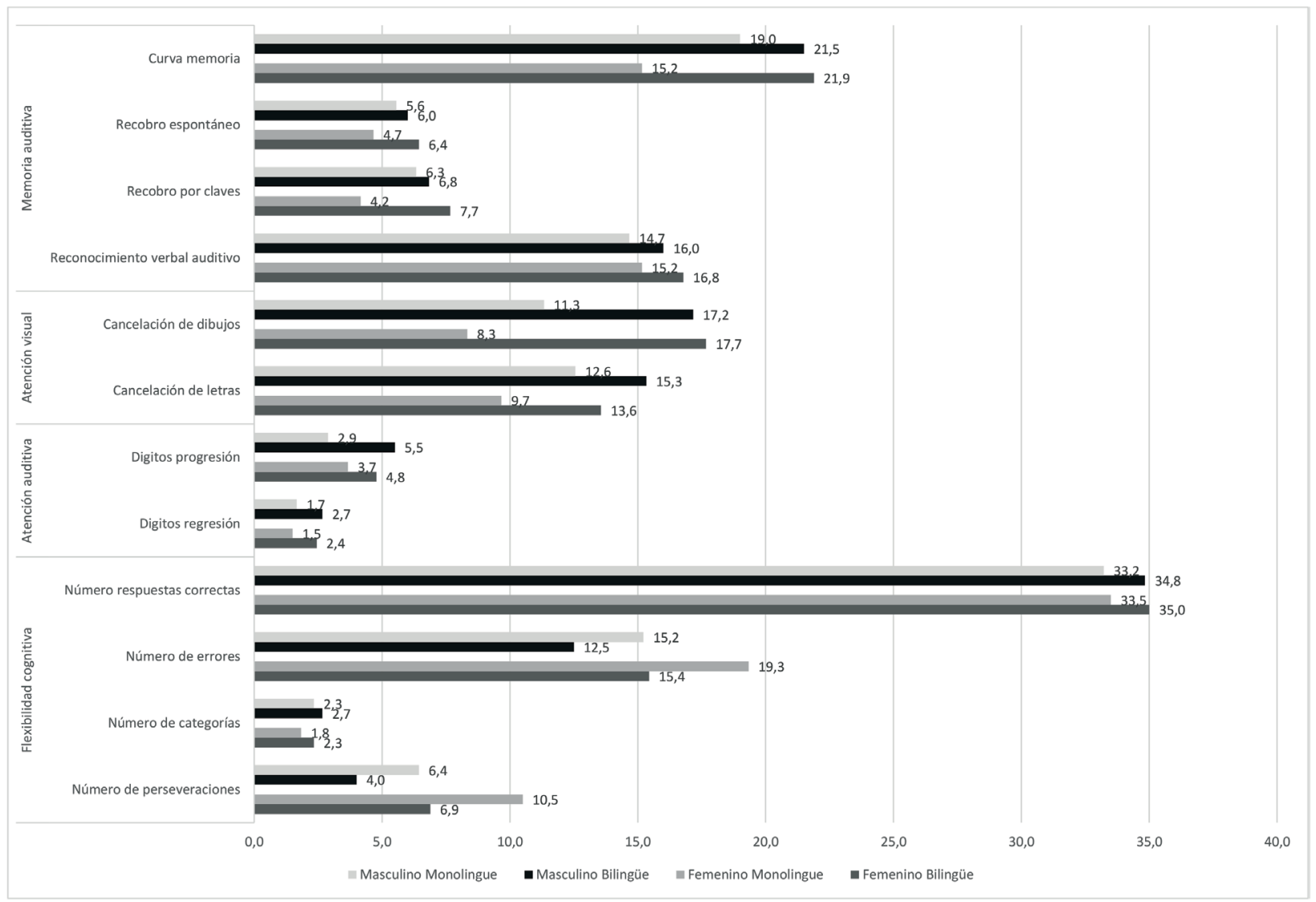

Fuente: elaboración propia.

Respecto a la memoria auditiva, en la curva de memoria de participantes de sexo femenino, el grupo de monolingües fue el que tuvo una menor puntuación media, mientras que el grupo de bilingües tuvo la mayor puntuación media, con una diferencia porcentual de $44,3 \%$ entre ambas puntuaciones. En los participantes de sexo masculino, también el grupo bilingüe tuvo la mayor puntuación media, $13,2 \%$ superior a la del grupo de monolingües. En el recobro espontáneo, en las participantes de sexo femenino, el grupo de monolingües fue el que tuvo una menor puntuación media, mientras que el grupo de bilingües tuvo la mayor puntuación media, con una diferencia porcentual de $38,1 \%$ entre ambas puntuaciones. En los participantes de sexo masculino, igualmente el grupo bilingüe tuvo la mayor pun- tuación media, $8 \%$ superior a la del grupo de monolingües. En el recobro por claves, en las participantes de sexo femenino, el grupo de monolingües fue el que tuvo una menor puntuación media, mientras que el grupo de bilingües tuvo la mayor puntuación media, una con diferencia porcentual de $84 \%$. En los participantes de sexo masculino, igualmente el grupo bilingüe tuvo la mayor puntuación media, 7,9\% superior a la del grupo de monolingües.

En el reconocimiento verbal auditivo, en las participantes de sexo femenino, nuevamente el grupo de monolingües fue el que tuvo una menor puntuación media, mientras que el grupo de bilingües tuvo la mayor puntuación media, con una diferencia porcentual de 10,6\%. En los participantes de sexo 
masculino, igualmente el grupo bilingüe tuvo la mayor puntuación media, 9,1\% superior a la del grupo de monolingües.

Adicionalmente, respecto a las participantes de sexo femenino, se determinó que existían diferencias estadísticamente significativas entre bilingües y monolingües en la curva de memoria, el recobro espontáneo y el recobro por claves. Por su parte, para los participantes de sexo masculino, no se hallaron diferencias estadísticamente significativas entre bilingües y monolingües en ninguna de las cuatro pruebas. Es importante destacar que, para la memoria auditiva, respecto a la curva de memoria, el recobro espontáneo y el recobro por claves, el porcentaje de diferencia entre bilingües y monolingües fue mucho mayor en las participantes de sexo femenino en comparación con los de sexo masculino.

Respecto a la atención visual, en la cancelación de dibujos, de las participantes de sexo femenino el grupo de monolingües fue el que tuvo una menor puntuación media, mientras que el de bilingües tuvo la mayor puntuación media, con una diferencia porcentual de $112 \%$ entre ambas puntuaciones. En los participantes de sexo masculino, también el grupo bilingüe tuvo la mayor puntuación media, un 51,5\% superior a la del grupo de monolingües. Respecto a la cancelación de letras, las monolingües fueron las que tuvieron una menor puntuación media, mientras que las bilingües lograron la mayor puntuación media, con una diferencia porcentual de 40,2\%. En los participantes de sexo masculino, igualmente el grupo bilingüe tuvo la mayor puntuación media, superior en 22,1\% a la del grupo de monolingües. Adicionalmente, se determinó que existían diferencias estadísticamente significativas entre las bilingües y las monolingües en la cancelación de dibujos. Por su parte, se determinó que no existían diferencias estadísticamente significativas entre los bilingües y los monolingües en ninguna de las dos pruebas. Es importante destacar, respecto a la atención visual, que la diferencia porcentual entre bilingües y monolingües fue de casi el doble en ambas pruebas, para las participantes de sexo femenino en comparación con los participantes de sexo masculino.

En atención auditiva, en los dígitos en progresión, el grupo de las monolingües fue el que tuvo una menor puntuación media, mientras que el grupo de las bilingües tuvo la mayor puntuación media, existiendo entre ambas puntuaciones una diferencia porcentual de 30,3\%. En los participantes de sexo masculino, también el grupo bilingüe tuvo la mayor puntuación media, un 90,4\% superior a la de los monolingües. En los dígitos en regresión, el grupo de las monolingües fue el que tuvo una menor puntuación media, mientras que el de las bilingües tuvo la mayor puntuación media, con una diferencia porcentual de $63 \%$ entre ambas puntuaciones. En los participantes de sexo masculino, igualmente los bilingües tuvieron la mayor puntuación media, un $60 \%$ superior a la del grupo de monolingües.

Además, en las participantes de sexo femenino, se determinó que no existían diferencias estadísticamente significativas entre bilingües y monolingües en ninguna de las dos pruebas. Por su parte, en los participantes de sexo masculino, se determinó que existían diferencias estadísticamente significativas entre bilingües y monolingües en las dos pruebas. Respecto a la atención auditiva, el porcentaje de diferencia entre bilingües y monolingües fue de casi el triple en la prueba de dígitos en progresión, para las participantes de sexo masculino en comparación con los participantes de sexo femenino.

En cuanto a la flexibilidad cognitiva, en el número de respuestas correctas, en las participantes de sexo femenino, el grupo de monolingües fue el que tuvo una menor puntuación media, mientras que las bilingües tuvieron la mayor puntuación media, existiendo entre ambas puntuaciones una diferencia porcentual de 4,5\%. En los participantes de sexo masculino, también el grupo bilingüe tuvo la mayor puntuación media, un 4,8\% superior a la del grupo de monolingües. En el número de errores, en las participantes de sexo femenino, el grupo de bi- 
lingües fue el que tuvo una menor puntuación media, mientras que el de monolingües tuvo la mayor puntuación media, con una diferencia porcentual de 20,1\% entre ambas puntuaciones. En los participantes de sexo masculino, igualmente el grupo monolingüe tuvo la mayor puntuación media, un 17,9\% superior a la de los bilingües.

En el número de categorías, el grupo de las monolingües fue el que tuvo una menor puntuación media, mientras que el de las bilingües tuvo la mayor puntuación media, existiendo entre ambas puntuaciones una diferencia porcentual de $27,3 \%$. En los participantes de sexo masculino, igualmente el grupo bilingüe tuvo la mayor puntuación media, siendo un $14,3 \%$ superior a la del grupo de monolingües. En el número de perseveraciones, en las participantes de sexo femenino, el grupo de bilingües fue el que tuvo una menor puntuación media, mientras que el grupo de monolingües tuvo la mayor puntuación media, existiendo entre ambas puntuaciones una diferencia porcentual de 34,4\%. En los participantes de sexo masculino, igualmente el grupo monolingüe tuvo la mayor puntuación media, siendo un $37,9 \%$ superior a la del grupo de bilingües.
Por último, en los participantes de ambos sexos, se determinó que no existían diferencias estadísticamente significativas entre bilingües y monolingües en ninguna de las dos pruebas. Es importante destacar que, para la prueba de flexibilidad cognitiva, el porcentaje de diferencia entre bilingües y monolingües para ambos sexos es muy similar en el número de respuestas correctas, número de errores y número de perseveraciones.

\section{Correlaciones entre Cl y memoria auditiva, atención y flexibilidad cognitiva}

En esta sección se analizó si existía correlación entre las pruebas $\mathrm{Cl}$ con las pruebas de memoria auditiva, atención y flexibilidad cognitiva, tanto para bilingües como para monolingües. Dado que inicialmente se había verificado que los resultados no seguían una distribución normal, se seleccionó el coeficiente de correlación de Spearman como estadístico de prueba. En la Tabla 4 se presentan el valor del coeficiente de correlación con su respectivo valor $p$. No obstante, los resultados mostraron que no existían correlaciones significativas (valor $p<0,05$ ) en ninguno de los casos.

Tabla 4. Correlaciones entre prueba de $\mathrm{Cl}$ con memoria auditiva, atención y flexibilidad cognitiva para bilingües y monolingües

\begin{tabular}{|c|c|c|c|c|c|}
\hline \multirow[b]{3}{*}{ Categorias } & \multirow[b]{3}{*}{ Variables } & \multirow{2}{*}{\multicolumn{2}{|c|}{$\begin{array}{c}\text { Bilingüe } \\
\mathrm{CI}\end{array}$}} & \multirow{2}{*}{\multicolumn{2}{|c|}{$\begin{array}{c}\text { Monolingüe } \\
\mathrm{Cl} \\
\end{array}$}} \\
\hline & & & & & \\
\hline & & C.C. & Valor $p$ & c.C. & Valor $p$ \\
\hline \multirow{4}{*}{ Memoria auditiva } & Curva de memoria & $-0,023$ & 0,935 & 0,367 & 0,178 \\
\hline & Recobro espontáneo & 0,122 & 0,664 & 0,203 & 0,468 \\
\hline & Recobro por claves & 0,307 & 0,266 & 0,144 & 0,608 \\
\hline & Reconocimiento verbal auditivo & 0,123 & 0,663 & 0,491 & 0,063 \\
\hline \multirow{2}{*}{ Atención visual } & Cancelación de dibujos & 0,319 & 0,247 & $-0,082$ & 0,771 \\
\hline & Cancelación de letras & 0,432 & 0,108 & $-0,027$ & 0,923 \\
\hline \multirow{2}{*}{ Atención auditiva } & Dígitos en progresión & $-0,144$ & 0,608 & 0,247 & 0,374 \\
\hline & Dígitos en regresión & 0,141 & 0,616 & 0,290 & 0,295 \\
\hline \multirow{4}{*}{ Flexibilidad cognitiva } & Respuestas correctas & 0,340 & 0,214 & 0,123 & 0,663 \\
\hline & Errores & $-0,085$ & 0,762 & 0,108 & 0,701 \\
\hline & Categorías & 0,298 & 0,280 & $-0,021$ & 0,941 \\
\hline & Perseveraciones & 0,073 & 0,796 & $-0,368$ & 0,177 \\
\hline
\end{tabular}

Fuente: elaboración propia. 


\section{Discusión}

La presente investigación intenta evidenciar el impacto de la exposición temprana al bilingüismo respecto en un contexto escolar, para lo cual se analizaron los diferentes factores que inciden en el fenómeno y se identificó su impacto en las funciones ejecutivas, a saber: atención, flexibilidad, memoria de trabajo.

Existe un aporte del bilingüismo aditivo, lo cual se evidencia en los resultados para la muestra bilingüe del presente estudio, específicamente en lo que tiene que ver con el desarrollo de las funciones cognitivas de atención y memoria de una manera importante. Los resultados evidenciaron un alto desempeño de los bilingües en las pruebas de memoria verbal auditiva, atención visual y atención auditiva, frente a niños monolingües, como lo muestran los estudios de Bialystok (2015; 2011), Cummins 2001), Ardila (2010) y Smekal (2014).

Pese a que Bialystok y Martin (2004) afirman que los efectos en este tipo de bilingües no son tan fuertes como los de un bilingüismo proficiente en ambas lenguas, tal afirmación no excluye que exista algún tipo de beneficio para el desarrollo cognitivo. Los datos encontrados muestran que efectivamente sí hay un impacto en las funciones cognitivas generales de los "aprendices de una segunda lengua". De alli puede derivar que en los resultados haya habido diferencias y desempeño superior de los bilingües en pruebas de memoria y atención, frente a los monolingües, pero no en cuanto a flexibilidad cognitiva, en la misma vía de lo descrito por Cummins (2001) y Ardila (2010), quienes refieren efectos positivos del bilingüismo aditivo en el desarrollo de habilidades académicas y destrezas cognitivas, cuando existen ciertos "umbrales" de exposición a la L2, lo que justificaría el desempeño superior de la población bilingüe de este estudio. Incluso Sánchez y Tembleque (1986), Janciauskas y Chang (2017) refieren que, por cada año que se retarde el aprendizaje de una L2, se va perdiendo motivación, de allí el mayor impacto de una exposición más temprana, sobre todo entre los 4 y los 8 años.
El desempeño de los bilingües fue superior en las categorias de memoria verbal auditiva (curva de memoria, recobro espontáneo, reconocimiento verbal auditivo y recobro por claves), donde, al parecer, la exposición temprana al bilingüismo tiene un impacto importante; sin embargo, en cuanto a la atención visual, solo en la cancelación de dibujos se obtiene mejor desempeño que en la cancelación de letras, y lo mismo sucede en cuanto a atención auditiva y memoria de trabajo, que tienen un desempeño superior en los bilingües en dígitos en progresión, pero sin diferencias significativas en dígitos en regresión, aunque ello no afecta el puntaje de desempeño general en estas pruebas.

Respecto a la atención, si bien Bialystok (2001), Bialystok y Martin (2004), Smekal (2014) y Carrada (2014) refieren que uno de los mayores efectos del bilingüismo reside en el proceso atencional selectivo, en este estudio se encuentran resultados variables, tal vez debido a que el efecto en las etapas iniciales se encuentra apenas en consolidación, de forma que los bilingües mostraron frente a los monolingües un alto desempeño en la subprueba de cancelación de dibujos, pero un desempeño no significativo en la cancelación de letras, aunque también pudiera deberse a que apenas se ha empezado el proceso lectoescritor (letras).

Adicionalmente, no existen diferencias significativas en los desempeños en flexibilidad cognitiva para los casos y el grupo control. Tales resultados están bien fundamentados en el hecho de que las modificaciones relacionadas con el rendimiento de las funciones ejecutivas son directamente proporcionales al tiempo de exposición a la segunda lengua, como mencionan Bialystok y Barac (2012), Wong, Ying y O’Brien (2016) y Kroll y Bialystok (2014). Teniendo en cuenta que la exposición de la muestra de este estudio no supera los tres años, se evidencia una especie de efecto inicial, además del hecho de que dicha función ejecutiva no se ha desarrollado tan tempranamente (niños de 6 años). 
Los resultados evidencian que, para que aumente el impacto del bilingüismo de manera contundente sobre cada una de las funciones ejecutivas, se requerirá de un mayor grado de exposición a los entornos educativos bilingües de modo que influya más en las habilidades académicas y los procesos superiores de pensamiento (Bialystok, 2001; 2015; Cendoya, 2009; Bialystok y Martin (2004). Sin embargo, el presente estudio pudo comprobar un aporte inicial e incipiente al desarrollo de la mayor parte de las funciones ejecutivas evaluadas.

Estos hallazgos con respecto a la flexibilidad cognitiva de los grupos comparados tienden a ser similares a los encontrados en el estudio realizado en Colombia por Villamizar y Guevara (2014), en el que, incluso en población adolescente, no se encuentran diferencias entre la flexibilidad cognitiva de bilingües y monolingües -dato justificado en ese estudio en un bilingüismo tardío-, al igual que lo encontrado por Martínez y Henao (2006) en su evaluación de la flexibilidad cognitiva, sin que hubieran hallado diferencias estadísticas entre bilingües y monolingües. Se debe tener en cuenta que en ambas investigaciones el tamaño de la muestra también era limitado.

Respecto al sexo, factor que se analizó en cuanto al desempeño por subpruebas, se evidencia en la muestra obtenida la influencia de las diferencias biológicas asociadas al aprendizaje de una segunda lengua. Particularmente, las niñas bilingües tuvieron un mejor desempeño en lo relacionado con memoria verbal auditiva y atención visual, comparado con el de los varones bilingües, los cuales, sin embargo, tuvieron mejor atención auditiva. Tales desempeños pueden ser explicados por la manera como se realiza el procesamiento global para el sexo femenino, por su tendencia a usar de manera simultánea ambos hemisferios en el procesamiento de la información, mientras que los varones suelen ser más lateralizados. Incluso tales diferencias están explicadas también por la mejor interacción entre las niñas que la que se da entre los niños en el proceso de aprendizaje (Jiménez, 2010).
No obstante, los resultados encontrados en la presente investigación por sexo van en contravía de lo encontrado por Carrada (2014), pues allí los varones tuvieron mejor desempeño en atención visual, aunque que en dicho estudio se evaluaron niños de 5 a 12 años, lo cual pudiese explicar las diferencias en los resultados en virtud de la edad y el mayor grado de exposición a un segundo idioma (véase igualmente Galarza, Padilla y Bonilla, 2005).

Por último, otro factor incidente en el desempeño de los niños bilingües es el estrato socioeconómico, que influye incluso en el desarrollo de las funciones ejecutivas, teniendo en cuenta que el 100\% de la muestra elegida para los bilingües correspondió a los estratos 4 a 6 . En cuanto al funcionamiento cognitivo, este se encuentra ampliamente documentado por Arán Filippetti (2012) y Matute, Sanz, Gumá, Rosselli y Ardila (2010) -citados por Montoya y Betancur (2017)-, en cuyos estudios la edad predice las ejecuciones en tareas que evalúan atención y memoria y donde se afirma que los niños de estratos bajos presentan un peor perfil cognitivo, a menor edad, que los de estrato medio. Arán Filippetti (2012) ha establecido que el desarrollo cognitivo evidenciado en las pruebas objetivas se explica por la presencia de estimulación cognitiva en el hogar. Otros estudios (Noble, McCandliss y Farah, 2007; Noble, Norman y Farah, 2005; y Matute et al., 2009) citados por Barac y Bialystok (2012) encuentran que el nivel educativo de los padres es el componente más asociado al funcionamiento cognitivo, lo cual se evidenció en el desempeño de las pruebas aplicadas a lo largo de esta investigación, donde el 95\% de los padres de los niños bilingües tenía formación universitaria y de posgrado versus los niños monolingües, hijos de padres con formación bachiller en un 90\% de los casos.

Barac y Bialystok (2012) evidencian que -tal como lo mencionan Noble et al. (2007); Arán (2012), Noble, Wolmetz, Ochs, Farah y McCandliss (2006), citados por Montoya y Betancurt (2017)- existe una correlación entre la instrucción de los padres y el des- 
empeño en tareas de memoria verbal y de trabajo, siendo el 95\% de los padres de niños bilingües de alta instrucción académica (posgrado). De la misma forma, Matute et al. (2009) evidencian que se encuentra mejor desempeño mnésico en alumnos de instituciones educativas privadas cuyos padres tienen un nivel educativo alto y que, tal como lo muestra la teoría, es imposible aislar del bilingüismo el efecto del estrato socioeconómico (Calvo y Bialystok, 2014).

Como mencionan Ardila, Rosselli, Matute y Guajardo (2005), las pruebas neuropsicológicas son muy sensibles a variables culturales y educativas y el desarrollo cognitivo se correlaciona con el estatus socioeconómico Matute et al. (2009), Carrada, (2014).

\section{Conclusión}

El grupo de niños bilingües mostró más alto desempeño que los monolingües en las subpruebas de la evaluación neuropsicológica infantil (ENI) para memoria verbal auditiva, atención auditiva y una de las dos subpruebas de atención visual, sin diferencias significativas en los desempeños de flexibilidad cognitiva. Existe mejor desempeño de las niñas bilingües frente a niños bilingües y niñas y niños monolingües para las pruebas de memoria verbal auditiva y atención visual. Por último, se evidencia la correlación entre estrato socioeconómico y escolaridad de los padres de estrato 4, 5 y 6, para bilingües con padres de formación universitaria y posgradual en un $90 \%$, versus los niños monolingües pertenecientes a estrato 3, con padres bachilleres en su mayoría. No hubo correlación entre los resultados obtenidos para prueba de coeficiente intelectual y el desempeño de los bilingües en las pruebas, lo que permite afirmar que el efecto del coeficiente no tuvo injerencia en tales resultados, sino que podemos atribuir las diferencias directamente al proceso de exposición a una segunda lengua.

Como limitaciones y posibles nuevos avances a partir de la presente investigación se debe tener en cuenta que el tamaño de la muestra fue más bien escaso, además que, en el contexto de un estrato socioeconómico alto, se vuelve más difícil encontrar niños monolingües, pues gran parte de la educación de alta calidad y costo es netamente bilingüe e incluso multilingüe. De igual forma, se sugieren estudios de tipo longitudinal para identificar el curso del desarrollo de las funciones ejecutivas para los bilingües aditivos.

Como conclusión final, es claro que los datos de este estudio muestran de manera concordante con los antecedentes teóricos el efecto del bilingüismo en el desarrollo de las funciones ejecutivas, en especial de la memoria verbal auditiva, y de manera parcial en la atención tanto visual como auditiva, lo cual fundamenta el posterior desarrollo de las habilidades académicas y funciones cognitivas superiores.

\section{Referencias}

Arango, O., Puerta, I., Montoya, P.y Sánchez, J. (2013). Predictores psicolingüísticos de la adquisición y aprendizaje del inglés como segunda lengua. Avances en Psicología Latinoamericana, 31(3), 546-555. Recuperado de https://revistas.urosario.edu.co/index.php/apl/article/view/2228

Ardila, A. (2010). Ventajas y desventajas del bilingüismo. Forma y Función, 25(2), 99-114.

Ardila, A., Rosselli, M., Matute, E y Guajardo, S. (2005). La influencia del nivel educativo de los padres en el desarrollo de las funciones ejecutivas. Develomental Neuropsychology, 28(1), 539-560. Recuperado de https:// revistas.unal.edu.co/index.php/formayfuncion/article/view/39833/ 
Barac, R. y Bialystok, E. (2012). Bilingual effects on cognitive and linguistic development: Role of language, cultural background an education. Child development, 2(2), 413-422. DOI: https://dx.doi.org/10.1111/j.14678624.2011.01707.x

Bialystok, E. (2015). Bilingualism and the development of executive function: the role of Attention. Child Development Perspectives, 9(2),117-121. https://dx.doi.org/10.1111/cdep.12116

Bialystok, E. (2011). Coordination of executive functions in monolingual and bilingual children. Journal of Experimental Child Psychology. 110, 461-468. DOI: https://doi.org/10.1016/j.jecp.2011.05.005

Bialystok, E. (2001). Bilingualism in Development: Language, Literacy, and Cognition. Nueva York: Cambridge University Press.

Bialystok, E. (2000). Language processing in bilingual children. Nueva York: Cambridge University Press.

Bialystok, E.y Barac, R. (2012). Emerging biligualism: Dissociating advantages for metalinguistic awareness and executive control. International Journal of Cognitive Science, 1(122), 67-73. DOl: http://dx.doi.org/10.1016/j. cognition.2011.08.003

Bialystok, E. y Feng, X. (2009). Language proficiency and executive control in proactive interference: Evidence from monolingual and bilingual children and adults. Brain and Language, 109(122), 93-100. DOI: https:// doi.org/10.1016/j.bandl.2008.09.001

Bialystok, E. y Martin, M. (2004). Attention and inhibition in bilingual children: evidence from the dimensional change card sort task. Developmental Science, 7(3), 325-339. DOI: https://doi.org/10.1111/j.14677687.2004.00351.x

Bialystok, E., Morales, J. y Calvo, A. (2013). Working memory development in monolingual and bilingual children. Journal of Experimental Child Psychology, 114(2), 187-202. DOI: https://doi.org/10.1016/j.jecp.2012.09.002

Calvo, A. y Bialystock, E. (2014). Independent effects of bilingualism and socio economic status on language ability and executive functioning. International Journal of Cognitive Science, 130(1), 278-288. DOI: https:// dx.doi.org/10.1016/j.cognition.2013.11.015

Calvo, N., Ibáñez, A. y García, A. (2016). The impact of biligualism on working memory: A null effect on the whole may not be so on the parts. Frontiers in Psychology, 7(285), 1-4. DOI: https://doi.org/10.3389/fpsyg.2016.00265

Carrada, M. (2014). La evaluación de la atención en niños escolarizados: el efecto de la edad, el género y el contexto sociocultural. Diálogos, 4(2), 139-156.

Cintrón-Valentín, M. y Ellis, N. (2016). Salience in second language acquisition: Physical form, learner attention, and instructional focus. Frontiers in Psychology, 7(1284), 1- 21. DOI: https://doi.org/10.3389/fpsyg.2016.01284

Cummins, J. (2001). ¿Qué sabemos de la educación bilingüe? Perspectivas psicolingüísticas y sociológicas. Revista de Educación, 325(1), 37-61. Recuperado de http://redined.mecd.gob.es/xmlui/bitstream/handle/11162/76005/008200230392.pdf 
Fandiño-Parra,Y.J., Bermúdez-Jiménez, J. R.y Lugo-Vásquez, V. E. (2012). Retos del Programa Nacional de Bilingüismo. Colombia Bilingüe. Educación y Educadores, 15(3), 363-381. DOI: https://doi.org/10.5294/edu.2012.15.3.2

Galarza, J., Padilla, A. y Bonilla, J. (2005). Evaluación neuropsicológica de una muestra de niños de 5 a 12 años con instrucción escolar bilingüe. Interação em Psicologia, 9(1), 125-130. DOI: http://dx.doi.org/10.5380/psi.vgi1.3292

Gorman, B. (2012). Relationships between vocabulary size, working memory, and phonological awareness in spanish speaking english language learners. American Journal of Speech Language Pathology, 21(1), 109123. DOI: https://doi.org/10.1044/1058-0360(2011/10-0063)

Instituto Cervantes (s.f.). Dependencia de campo. En Diccionario de términos clave de ELE. cvc.cervantes.es. Recuperado de https://cvc.cervantes.es/ensenanza/biblioteca_ele/diccio_ele/diccionario/dependencia.htm

Janciauskas, M. y Chang, F. (2017). Input and age-dependent variation in second language learning: A connectionist account. Cognitive Science. A Multidisciplinary Journal, 42, 519-554. DOI: https://doi.org/10.1111/cogs.12519

Jiménez, E. (2010). El factor género en el proceso de adquisición de lenguas: Revisión Crítica de los Estudios Interdisciplinares, 1(8), 1-20. Recuperado de http://www.linred.es/articulos_pdf/LR_articulo_30042010.pdf

Jung, I. y López, L., comps. (2003). Abriendo la escuela: Lingüística aplicada a la enseñanza de las lenguas. Madrid: Morata.

Kroll, J.y Bialystok, E. (2014). Understanding the consequences of bilingualism for language processing and cognition. Journal of Cognitive Psychologie, 25(5), 1-22. DOI: https://dx.doi.org/10.1080/20445911.2013.799170

Kuhl, P., K. (2010). Brain mechanisms in early language acquisition. Neuron Review, 67, 713-727. DOI: https:// dx.doi.org/10.1016/j.neuron.2010.08.038

Martínez, M. y Henao, G. (2006). Desempeño de la función ejecutiva de niños expuestos a ambientes de enseñanza bilingües. Revista Electrónica de Educación Psicoeducativa, 10(4), 513-528. Recuperado de http:// www.investigacion-psicopedagogica.org/revista/articulos/10/espannol/Art_10_123.pdf

Matute, E., Sanz, A., Gumá, E., Rosselli, M.y Ardila, A. (2009). Influencia del nivel educativo de los padres, el tipo de escuela y el sexo en el desarrollo de la atención y la memoria. Revista Latinoamericana de Psicología, 41(2), 257-276. Recuperado de http://www.redaly c.org/articulo.oa?id=80511496006

Mejía, A. M., López-Mendoza, A. y Peña, B., comps. (2011). Bilingüismo en el contexto colombiano: iniciativas y perspectivas en el siglo XXI. Bogotá: Universidad de los Andes.

Montoya-Zuluaga, P. A. y Betancur-Arias, J. D., comps. (2017). Hacia un concepto multifactorial del aprendizaje y la memoria. Aproximaciones neuropsicopedagógicas (vol. I). Medellín: Universidad Católica Luis Amigó.

Mora, J. (2012). Estilos cognitivos en niños y niñas en condición de exposición al bilingüismo. Tesis de Maestría en Desarrollo Infantil, Universidad de Manizales, Colombia.

Morales, J., Calvo, A. y Bialystok, E. (2013). Working memory development in monolingual and bilingual children. Journal of Experimental Child Psychology, 114(2), 187-202. DOI: https://doi.org/10.1016/j.jecp.2012.09.002 
ISSN 0123-1294 | e-ISSN 2027-5358 | Educ.Educ. Vol. 22. No. 2 | Mayo-agosto de 2019 | pp.167-186.

Universidad de La Sabana | Facultad de Educación

Poulin-Dubois, D., Blaye, A., Coutya, J. y Bialystok, E. (2011). The effects of biligualism on toddlers' executive functioning. Journal of Experimental Child Psychology, 108(1), 567-579. DOI: https://dx.doi.org/10.1016/j. jecp.2010.10.009

Sánchez, A. (2013). Bilingüismo en Colombia. Documentos de Trabajo sobre Economía Regional, 191, 1-47. Recuperado de http://www.banrep.gov.co/docum/Lectura_finanzas/pdf/dtser_191.pdf

Sánchez, M. y De Tembleque, R. (1986). La educación bilingüe y el aprendizaje de una segunda lengua: sus características y principios fundamentales. Infancia y Aprendizaje, 9(33), 3-25. Recuperado de https://dialnet. unirioja.es/servlet/articulo?codigo $=2926342$

Sattler, J. M. (2003). Evaluación infantil: aplicaciones conductuales y clínicas. México: Manual Moderno.

Smekal, E. (2014). Evaluación de la atención selectiva en niños bilingües. En María Inés Rubi et al., Los vínculos interpersonales en educación y aprendizaje. XV Jornadas Trasandinas de Aprendizaje. La Plata: Trasandinas Libros.

Villamizar, G.y Guevara, E. (2013). Bilingüismo y desempeño de las funciones ejecutivas en adolescentes de colegios bilingües y no bilingües. Revista de Pedagogía, 34(94- 95), 35-58. Recuperado de http://www.redaly c. org/articulo.oa?id=65932613006

Wong, B., Ying, B. y O’Brien, B. (2016). Neuroliguistics: Structure, function, and conectivity in the bilingual brain. Biomedic Research International, 9, 1-22. DOI: http://dx.doi.org/10.1155/2016/7069274 